Henri Rodrigue Njengoué Ngamaleu

Université de Yaoundé 1

(Cameroun)

Céline Dang Olinga

Université de Yaoundé 1

(Cameroun)

\section{Aspirations socioprofessionnelles et stratégies de développement de carrière chez les enseignements du secondaire au Cameroun}

Socio-professional aspirations and career development
strategies among high school teachers in Cameroon

doi: $10.18162 / f p .2018 .438$

\section{R \\ ésumé}

Le présent article s'intéresse aux stratégies de développement de carrière des enseignants du secondaire. Il explore leurs aspirations socioprofessionnelles en tenant compte de I'incertitude qui caractérise le système éducatif au Cameroun. L'échantillon est constitué de 289 participants ayant rempli des échelles à sept niveaux suivant le modèle de Likert. Ils se montrent intéressés par les nominations aux postes de responsabilité, le détachement dans d'autres administrations, les affaires et la politique. Le renforcement des capacités et surtout l'implication au travail s'avèrent des stratégies moins favorables au développement de carrière, contrairement à l'engagement en politique et au recours aux réseaux relationnels influents.

Mots-clés

Aspirations socioprofessionnelles, développement de carrière, enseignants du secondaire, Cameroun

Abstract

Abstract

This paper focuses on secondary school teachers' career development strategies. It explores thereby their social and professional aspirations by taking into consideration the level of uncertainty which characterized the educational system in Cameroon. The sample is constituted of 289 participants who filled 7-point Likert scales. They show themselves interested in nominations at high positions, transfer in others administrations, business and politics.

The capacity building and mostly professional commitment are less efficacy career development strategies them political engagement and the using of influential relational networks.

\section{Keywords}

Socio-professional aspirations, career development, high school teachers, Cameroon.

\section{Introduction}

L'expression «faire carrière » renvoie à la progression d'un individu dans sa vie professionnelle,suivantdesétapessordonnant généralement dans un sens positif, d'une moindre à une plus grande qualification (Guichard et Huteau, 2007). Le contexte communautaire, sociétal et culturel est fondamental pour comprendre le sens d'une action, d'un projet ou d'une carrière (Young, Marshall et Valach, 2007; Young et Valach, 2000, 2006). D'une culture à l'autre, les systèmes d'interprétation de la carrière diffèrent. Au sein d'un contexte social et culturel, elle constitue une construction et une représentation déterminées d'actions dans le long terme. La culture est envisagée comme un processus réflexif, partagé, interactif et intergénérationnel portant sur les actions, les projets et les carrières (Young et al., 2007). C'est dans cette perspective que l'autodétermination, autrement l'agentivité de l'enseignant du secondaire camerounais, est envisagée en rapport avec le développement de sa carrière, dans un environnement socioadministratif dont nous décrirons les irrégularités.

\section{Agentivité humaine et développement de carrière}

Depuis les dernières décennies du $\mathrm{XX}^{\mathrm{e}}$ siècle, le concept de carrière est au cœur des théories et pratiques de lorientation scolaire et professionnelle en Amérique du Nord et dans la plupart des pays anglo-saxons. L'un des cadres théoriques dans lesquels ces pratiques s'enracinent est celui de la théorie sociale cognitive (TSC) de Bandura $(1989,1994)$ qui postule un déterminisme triadique entre la personnalité, le comportement et l'environnement. La TSC relève d'une psychologie de la compétence et met l'accent sur l'agentivité humaine, autrement dit la capacité d'autodirection de l'être humain. En cela, Bandura (1977, 1997, 2003) soutient que l'être humain est à la fois produit et producteur de son environnement. Lent, 
Brown et Hackett $(1994,2000)$ se sont servis des fondamentaux de la TSC de Bandura afin de cerner le développement de carrière : il s'agit de la théorie sociale cognitive de l'orientation scolaire et professionnelle (TSCOSP) (Lent, 2008). Dans la langue d'origine, les auteurs parlent de « Social Cognitive Theory of Career Development» (Lent, 2005). Cette théorie propose une compréhension du choix professionnel et du développement de carrière en convoquant les croyances d'efficacité personnelle, les attentes de résultats, les buts et les obstacles (contraintes environnementales).

En matière d'autodétermination, l'autoefficacité renvoie aux jugements que font les individus à propos de leurs capacités à atteindre des niveaux de performance définis (Bandura, 2003). Les attentes de résultats ont trait aux croyances personnelles relatives aux conséquences et aux résultats de la réalisation de comportements particuliers. Les activités que les personnes choisissent de réaliser ou d'éviter sont en grande partie déterminées par les sentiments d'efficacité personnelle et les attentes de résultats. Pour ce qui concerne les buts personnels, Bandura (1986) les présente comme l'intention qu'a une personne de s'engager dans une activité précise pour atteindre un objectif particulier. En se fixant des buts, les personnes contribuent à organiser, à diriger, et à renforcer leur propre comportement, et cela même sur de longues périodes et en l'absence de renforcements externes. Les buts sont de ce fait déterminants dans l'expression de l'agentivité personnelle, dans la réalisation de projets d'études ou professionnels. Le choix professionnel et le développement de carrière sont envisagés comme un processus dynamique et continu. Dans le cadre de la TSCOP, le choix et le parcours professionnel est un processus interactif qui tient de la réceptivité de l'individu à l'environnement.

Pour l'enseignant du secondaire au Cameroun, l'environnement dans lequel il développe sa carrière peut renvoyer au système éducatif, en tant qu'il est géré d'après les modes de gouvernance administratifs plus généraux. Il importe de s'appesantir sur les caractéristiques de cet environnement marqué par l'absence de respect et d'application scrupuleuse des textes (Ngon, 2015). L'enseignant interagit avec un environnement socioprofessionnel qui favorise des dysfonctionnements relatifs aux usages informels, illicites et illégaux, mais revêtant tout de même le statut de normes, du fait de leur pouvoir régulateur de comportements. L'ajustement des acteurs tient dans ce cas compte aussi bien des opportunités de développement de carrière prévues par les textes que de celles qui relèvent de la malgouvernance du système éducatif, notamment la gestion décriée des ressources humaines des corps concernés. On peut alors s'intéresser à la relation entre les aspirations socioprofessionnelles et les stratégies de développement de carrières qu'ils mobilisent en conséquence.

Les aspirations sont des buts à long terme (distal goal mechanisms) qui organisent les comportements de l'individu, notamment ceux liés aux études, et ce, même dans un contexte où les renforcements immédiats sont absents (Rasheed Ali et Saunders, 2008). O’Brien (1996) indique par exemple que les aspirations professionnelles sont définies par le degré avec lequel les individus aspirent à des postes de leadership et désirent poursuivre leur éducation tout au long de leur carrière. Les perceptions de compétence, autrement dit l'autoefficacité de Bandura (2003), sont associées à la motivation autodéterminée qui, à son tour, prédit positivement les aspirations professionnelles (Litalien et Guay, 2010). La motivation orientant l'organisme vers un but tout en déterminant l'intensité et la persévérance du comportement, si l'on souhaite examiner les stratégies que les enseignants du secondaire adoptent, en fonction de leurs aspirations, pour assurer le développement de leur carrière, il faut effectuer une exploration empirique. 


\section{L'environnement culturel du système éducatif camerounais}

Le système éducatif camerounais appartient à un macro-système, au sujet duquel Blundo et van Beek (2002) soutiennent la double thèse de la personnalisation de l'accès à l'administration et la privatisation informelle des institutions. La lutte contre la corruption prend toutes les allures du combat contre l'hydre à sept têtes au Cameroun (Titi Nwel, 1999, 2009; Transparency International, 2007). La Commission nationale anti-corruption déclare d'ailleurs que « la corruption semble la règle et l'intégrité l'exception » (CONAC, 2011). Elle cite l'éducation parmi les secteurs gangrénés par ce fléau.

À la veille de la rentrée scolaire 2015-2016, le bureau Cameroun de la Fondation Friedrich Ebert a organisé une conférence sous le thème «Éducation et corruption : comment combattre la corruption dans le système éducatif au Cameroun? ». Il en ressort le constat suivant : «Le Cameroun a remporté le prix du pays le plus corrompu de la planète à deux reprises, entre 1988 et 1999 dans le classement de l'indice de perception de la corruption de Transparency International. Dans un environnement comme celui-là, tout s'achète et se vend : l'élève ou l'étudiant achète les notes pour passer en classe supérieure, ensuite il achète le concours pour entrer dans une grande école, puis il achète les notes pour sortir de l'école et être affecté dans une grande métropole, et enfin il verse des prébendes pour être nommé plus tard. À son tour, il exige aux jeunes élèves de faire pareil et tout le système est infesté par ce cercle vicieux » (Friedrich-Ebert-Stiftung, 2015).

LaCONAC (2010,p. 56) citele « monnayage des recrutements, promotions, affectation et nominations » en tête de liste parmi les «manifestations de la corruption dans le secteur de l'éducation », et parmi les « causes de la corruption », elle fait mention, entre autres, du «non-respect du profil de carrière » Les causes et les manifestations de la corruption dans le secteur éducatif sont d'ordre politique et institutionnel. L'absence de transparence, d'équité et de justice dans le processus de traitement du personnel des administrations chargées de la gestion du secteur éducatif sont décriées et confirment bien que le développement de carrière de l'enseignant ne relève pas exclusivement de la régulation formelle du management de l'éducation. Il est d'ailleurs relevé que la gestion des carrières fait l'objet d'un « marché », du fait de la centralisation excessive de l'administration (CONAC, 2010, p. 57).

Ndongmo et Bayang (2015) soutiennent qu'en matière de gestion des ressources humaines du secteur éducatif, les affectations, les mutations et les nominations s'obtiennent en payant tout simplement. "Tous les postes d'administration sont "marchandables" et les prix sont connus. Ceux qui s'y connaissent vous abordent pour vous proposer ce marché salvateur pour votre carrière [...] l'achat des postes est devenu monnaie courante dans le secteur éducatif» (p. 45-46). Les auteurs mentionnent également qu'en dehors du monnayage comme moyen de promotion, « les relations comptent aussi énormément. Ainsi, on est nommé parce qu'on a une relation au ministère ou dans les autres instances décisionnelles importantes ». Il s'agit de ce que Njengoué Ngamaleu (2015) a essayé de conceptualiser en parlant de psychologie des relations sociales en contexte camerounais. Djateng (2012, p. 29-30) relève, par exemple, que les épouses des personnes influentes dans l'échelle politico-administrative du système accèdent facilement aux postes de responsabilité afin d'échapper aux contraintes liées aux obligations d'un « simple professeur des salles de classe ». 
Un statut particulier des corps des fonctionnaires de l'Éducation nationale existe pourtant depuis 2001. Il aménage une esquisse de profil de carrière censée régir les promotions. Djateng (2012) constate que dix ans après, le texte demeure sans application et il ajoute que le ministère de l'Éducation secondaire et ses structures déconcentrées "s'apparentent aujourd'hui à de vastes comptoirs commerciaux » (p. 29). Comme résultat, « on observe sur le terrain des personnes qui passent six mois au poste de surveillant général, un an à celui de censeur et puis font un grand saut l'année suivante comme chef d'établissement. [...] Le poste de chef d'établissement est très couru. Les prétendants se recrutent à des niveaux très divers des administrations scolaires. Même les Inspecteurs Nationaux, ceux-là qui sont parvenus au grade prestigieux de sous-directeur de l'administration centrale, retournent se faire nommer chef d'établissement, un grade moins prestigieux sur le plan protocolaire ou de la préséance » (p. 30).

Une autre manière de faire carrière consiste pour l'enseignant, aussi aberrant que cela puisse paraitre, à s'absenter ou à carrément abandonner le poste, sans que le salaire soit suspendu. Les concernés y parviennent en usant de stratagèmes qui impliquent les responsables hiérarchiques. Ils couvrent leurs protégés, en contrepartie d'une rémunération régulière convenue, et leur donnent la possibilité de recruter des vacataires qui interviennent dans leurs salles de classe en leur absence. Lorsque les contrôles sont annoncés et que la présence physique des absents irréguliers est requise pour un recensement physique du personnel, ils sont discrètement informés et/ou couverts, par la hiérarchie complice, et conservent ainsi leur statut de salarié de la fonction publique. Djateng (2012, p. 33) indique à cet effet que des enseignants résidant au Canada, aux États-Unis ou au Gabon sont, sur le plan administratif, paradoxalement présents à leur poste de travail. Ils s'arrangent à payer le vacataire qui les supplée et multiplient leurs sources de revenus en exerçant d'autres activités d'ailleurs.

Cette recherche d'un meilleur pouvoir d'achat et l'amélioration des conditions de vie font constater ce que Omgba (2015, p. 194) appelle la «forte mobilité » des agents de l'administration publique camerounaise. Un de ses aspects a trait à l'aspiration indéniable des enseignants, affectés dans les zones rurales, à s'évertuer à les quitter pour des postes dans les établissements des zones urbaines, ceux des grandes villes notamment. « La corruption et le népotisme aidant, la mobilité se fait encore de manière plus facile, voire banale. Les enseignants sont affectés à tout moment de l'année, sans tenir compte des besoins de l'établissement scolaire d'où ils partent ni de ceux de l'école de leur destination » (p. 194).

Ils peuvent à cet effet choisir délibérément la localité et l'établissement où ils vont en fonction de son prestige, de sa proximité avec le domicile, ou même du nombre élevé d'enseignants dans la discipline enseignée, ce qui, selon Omgba (2015, p. 194), « augmente les chances de l'enseignant concerné d'avoir le moins de travail possible».

Une autre possibilité consiste à se faire affecter ou promouvoir de manière à occuper des fonctions administratives : un bureau de fonctionnaire est désormais le lieu permanent de travail de l'enseignant qui cesse de fréquenter les salles de classe. Il s'agit dans le langage courant « d'abandonner la craie ». Les charges administratives recèleraient plus de privilèges, pour moins de contraintes perçues en termes de charge de travail. Le temps ainsi dégagé permet de vaquer à d'autres occupations et de multiplier les sources de revenus. "Cette mobilité peut aussi prendre la forme d'une promotion à des responsabilités administratives, lesquelles peuvent également avoir lieu tout au long de l'année. Ils sont nombreux les enseignants qui se voient promus aux postes de surveillant général, censeur, ou même chef d'établissement, indépendamment de leur ancienneté ou de toute autre condition requise par la réglementation » (p. 195). 
Le détachement est également une forme de promotion prisée. Il s'agit de la position de l'enseignant placé hors de son corps d'origine, mais continuant à bénéficier, dans ce corps, de ses droits à l'avancement et à la retraite. L'enseignant est ainsi retiré à son administration d'origine pour être inséré dans une autre, n'ayant le plus souvent rien à voir avec l'éducation, où il fait désormais carrière, et ne retourne que rarement à son administration d'origine. Les enseignants du secondaire sont de ce fait présents dans les autres administrations.

Leur développement de carrière, sans doute autant que celui d'autres agents de la fonction publique (Njengoué Ngamaleu et Nkelzok Komtsindi, 2017), s'opère dans un environnement culturel et administratif qui amène à remettre en question l'autodétermination de l'individu en contexte d'incertitude. Dans une logique sociale cognitive, au sens de Bandura, le développement de carrière participe de l'agentivité humaine, en tant que capacité à construire sa propre trajectoire socioprofessionnelle. L'agent de la fonction publique développe un sens de l'efficacité en rapport avec les buts et objectifs qu'il se fixe, ses attentes de résultats, les opportunités et obstacles auxquels l'environnement le confronte. Dans les contextes d'Afrique noire, il importe d'insister sur ce caractère incertain de l'environnement, lequel s'avère complexe de fait. Les repères ne sont pas que formels, ce d'autant que la régulation sociale est, pour une part significative, assurée par les logiques et normes informelles, voire illicites ou illégales. Des faits et pratiques sont souvent documentés, comme dans les rapports précédemment mentionnés, sans pour autant faire l'objet d'une théorisation systématique des comportements d'autodétermination. C'est dans cette perspective que ce travail s'intéresse aux mécanismes cognitifs communs des individus ayant à transiger à la fois avec des normes formelles et informelles. L'objectif de ce travail est de décrire les relations entre les aspirations socioprofessionnelles des enseignants du secondaire camerounais et les stratégies de développement de leur carrière dans un environnement socioprofessionnel complexe.

\section{Méthodologie}

\section{Participants}

Un échantillon par convenance de 289 enseignants et enseignantes du secondaire a été constitué. En dehors des trois personnes qui nont pas donné de renseignement quant à leur sexe, 159 autres, soit $55,6 \%$ des effectifs valides, sont des hommes. La moyenne d'âge est de 36,87 $( \pm 7,407)$ ans; le plus jeune ayant 23 ans et le plus âgé, 57 ans. Les mariés sont les plus représentés dans l'échantillon (49\%) contre $21,5 \%$ de fiancés, $25 \%$ de célibataires et 4,5\% de divorcés. Le régime matrimonial dominant est la monogamie (51,3\%). Le nombre moyen de personnes à charge pour chaque répondant est de $3,96$ ( $\pm 2,528) ;$ le nombre le plus élevé étant de 14. Les dix régions du Cameroun sont diversement représentées dans l'échantillon. Les ressortissants de la région du Centre sont plus nombreux (37,8\%), suivis de ceux de l'Ouest $(19,4 \%)$, du Sud (10,6 \%) et de l'Est (8,1\%), parmi les plus représentés. L'ancienneté moyenne est de 8,02 $( \pm 6,122)$ ans; le plus jeune a moins d'une année de service alors que le plus ancien en compte 31 ans. En ce qui concerne les postes de responsabilité par nomination, $33,5 \%$ des répondants disent être concernés du fait qu'ils en occupent un au moment de l'enquête. Les disciplines enseignées sont variées et diversement représentées. Elles sont au nombre de 23 et recouvrent aussi bien les sciences pures, les sciences sociales et humaines, les langues, la comptabilité, les sciences 
économiques, l'économie sociale et familiale que la didactique. L'échantillon est majoritairement chrétien $(91,3 \%)$. Ils sont $50,2 \%$ et $7 \%$ à se dire respectivement engagés et très engagés sur le plan religieux. Le terrain ayant été fait dans la région du Centre, la plupart des enseignants rencontrés accidentellement sont en service au moment de l'enquête dans les établissements de la ville de Yaoundé et de ses environs. Toutefois, environ $10 \%$ des répondants sont en service dans d'autres régions du pays, même s'ils séjournent dans la ville de Yaoundé au moment de l'enquête.

\section{Échelles de mesure et test de cohérence interne}

Eu égard à la qualité de la gestion des ressources humaines dans l'enseignement secondaire camerounais, les aspirations socioprofessionnelles ont été opérationnalisées de manière empirique en quatre modalités. Il s'agit de : 1) l'intérêt pour les postes de nomination (items 1-6); l'intérêt pour le détachement dans des administrations autres que le ministère des Enseignements secondaires (items 711); les ambitions d'affaires (items 12-15); l'intérêt pour une carrière en politique (items 16-20). Pour ce qui concerne les stratégies de développement de carrière, également opérationnalisées de manière empirique, quatre autres modalités sont définies. Elles concernent $: 1)$ le renforcement des capacités (items 21-24); 2) l'implication au travail (items 25-34); 3) le militantisme politique (items 35-38) et 4) le réseau relationnel (items 39-43).

En dehors des variables sociodémographiques, de l'item 6 qui porte sur les différents postes de responsabilité auxquels aspirent les répondants et de l'item 11 qui les invite à indiquer par ordre de préférences trois administrations où ils aimeraient être détachés, tout en justifiant leur choix, tous les autres items correspondent à des échelles de mesure ordinale. Il s'agit précisément des échelles de Likert à 7 niveaux (Absolument faux-1-2-3-4-5-6-7-Absolument vrai). Le tableau 1 renseigne sur les valeurs de l'alpha de Cronbach basées sur les éléments normalisées pour toutes les dimensions mesurées. Elles ont été calculées au cours de la préenquête auprès de 33 enseignants du secondaire. En dehors des items portant sur les ambitions d'affaires, la cohérence interne est satisfaisante, voire excellente pour deux dimensions.

\section{Tableau 1}

Consistance interne des dimensions attitudinales.

\begin{tabular}{lcc}
\hline Dimension & Nombre d'items & $\boldsymbol{a}$ de Cronbach \\
\hline Intérêt pour les postes de nomination & 5 & 0,731 \\
Intérêt pour le détachement dans d'autres administrations & 4 & 0,735 \\
Ambitions d'homme d'affaires & 4 & 0,381 \\
Intérêt pour une carrière en politique & 5 & 0,940 \\
Renforcement des capacités & 4 & 0,640 \\
Implication au travail & 10 & 0,673 \\
Militantisme politique & 4 & 0,946 \\
Réseau relationnel & 5 & 0,667 \\
\hline
\end{tabular}




\section{Analyse des données}

Avant l'analyse des données proprement dite, l'item 1 est recodé parce que la formulation de la proposition qui s'y rapporte dans le questionnaire est inversée. Les outils des statistiques descriptive et inférentielle sont exploités dans le cadre de l'analyse des données collectées. Pour chaque item, la moyenne échantillonnale est déterminée ainsi que l'écart-type s'y rapportant. La médiane de l'échelle étant 4, les pourcentages de répondants réalisant des scores supérieurs à cette valeur, qui correspond théoriquement à la neutralité, indiquent le niveau d'adhésion à la proposition correspondante. Quant aux dimensions mesurées, les scores s'y rapportant s'obtiennent en additionnant pour chaque participant ses réponses aux items concernés. L'analyse peut également être faite de la même manière que celle des items pris individuellement. Les scores des dimensions sont toutefois traités comme de véritables variables numériques. La moyenne échantillonnale peut être calculée. Les pourcentages de participants réalisant des scores supérieurs à la moyenne théorique peuvent l'être également. Des tests de comparaison portant sur une moyenne permettent dans ce cas de déterminer la significativité des résultats dans une démarche inférentielle. La matrice de corrélation impliquant toutes les dimensions étudiées permet d'apprécier, pour chacune des aspirations socioprofessionnelles, le lien avec les stratégies de développement de carrière énumérées.

\section{Résultats}

La présentation des résultats commence par l'exposé des données relatives aux différentes aspirations socioprofessionnelles (tableau 2), ensuite celui des stratégies de développement de carrière (tableau 3). Le commentaire de la matrice de corrélations permettra de clarifier le lien entre les modalités des unes et des autres.

\section{Aspirations socioprofessionnelles}

Tableau 2

Dimensions des aspirations socioprofessionnelles.

\begin{tabular}{|c|c|c|c|c|c|c|c|c|}
\hline & Item & & Moy & Et & $\mathbf{t}$ & ddl & p & Adhésion (\%) \\
\hline \multirow{5}{*}{ 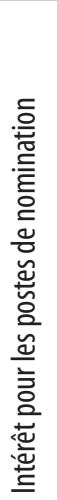 } & $q 1$ & $\begin{array}{l}\text { Je me contente d'être enseignant, je suis } \\
\text { indifférent aux postes de nomination. }\end{array}$ & 2,27 & 1,596 & $-18,341$ & 286 &, 000 & 14,6 \\
\hline & $q 2$ & $\begin{array}{l}\text { Achever une carrière n'ayant tenu que la craie } \\
\text { est une sorte de frustration. }\end{array}$ & 4,49 & 1,853 & 4,483 & 287 &, 000 & 56,9 \\
\hline & $q 3$ & $\begin{array}{l}\text { Aller de poste en poste dans la hiérarchie } \\
\text { administrative est une preuve de l'évolution } \\
\text { de la carrière. }\end{array}$ & 5,04 & 1,535 & 11,475 & 287 &, 000 & 75,0 \\
\hline & $q 4$ & $\begin{array}{l}\text { Ce sont les postes qui procurent pouvoirs, honneurs, } \\
\text { argents et avantages sociaux qui m'intéressent. }\end{array}$ & 4,58 & 1,787 & 5,494 & 285 &, 000 & 66,8 \\
\hline & $q 5$ & $\begin{array}{l}\text { Les collègues nommés aux postes convoités sont } \\
\text { en général les plus épanouis dans notre pays. }\end{array}$ & 4,93 & 1,629 & 9,659 & 287 &, 000 & 76,0 \\
\hline
\end{tabular}




\begin{tabular}{|c|c|c|c|c|c|c|c|c|}
\hline \multirow{4}{*}{ 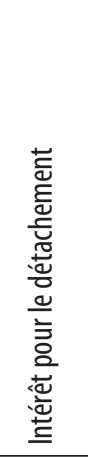 } & q7 & $\begin{array}{l}\text { Une nomination dans un autre département } \\
\text { ministériel me réjouirait. }\end{array}$ & 5,05 & 1,610 & 10,985 & 283 & 000 & 78,2 \\
\hline & q8 & $\begin{array}{l}\text { J'accepterai volontiers si la proposition d'être } \\
\text { détaché dans une autre administration m'était faite. }\end{array}$ & 5,46 & 1,271 & 19,343 & 284 &, 000 & 88,4 \\
\hline & q9 & $\begin{array}{l}\text { Les enseignants détachés dans d'autres } \\
\text { administrations sont plus épanouis que ceux } \\
\text { qui tiennent la craie. }\end{array}$ & 5,40 & 1,407 & 16,766 & 285 &, 000 & 85,7 \\
\hline & q10 & $\begin{array}{l}\text { Le détachement d'un enseignant du secondaire dans } \\
\text { une autre administration a toujours l'air } \\
\text { d'une promotion. }\end{array}$ & 5,60 & 1,132 & 23,753 & 283 &, 000 & 90,1 \\
\hline \multirow{4}{*}{ 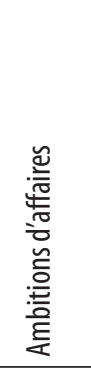 } & $q 12$ & $\begin{array}{l}\text { Je suis déterminé à créer des sources de revenus } \\
\text { autres que mon seul salaire. }\end{array}$ & 5,80 & 1,042 & 29,086 & 283 &, 000 & 93,7 \\
\hline & $q 13$ & $\begin{array}{l}\text { J'envisage de monter des projets qui emploient } \\
\text { des personnes. }\end{array}$ & 5,65 & 1,199 & 23,260 & 284 &, 000 & 90,2 \\
\hline & q14 & $\begin{array}{l}\text { Si mes activités parallèles réussissent, je suis capable } \\
\text { de me reconvertir définitivement dans les affaires. }\end{array}$ & 5,02 & 1,776 & 9,705 & 284 &, 000 & 71,9 \\
\hline & q15 & $\begin{array}{l}\text { J'aspire à gagner décemment ma vie en montant } \\
\text { et réalisant des projets d'affaires personnels. }\end{array}$ & 5,58 & 1,338 & 19,811 & 281 &, 000 & 87,9 \\
\hline \multirow{5}{*}{ 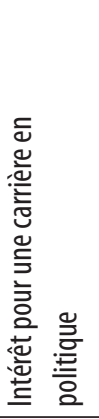 } & $q 16$ & Je suis intéressé par la politique. & 5,10 & 2,114 & 8,784 & 285 &, 000 & 71,3 \\
\hline & $\mathrm{q} 17$ & $\begin{array}{l}\text { J'aspire à faire partie des élites de ma localité par } \\
\text { mon engagement en politique. }\end{array}$ & 5,21 & 2,168 & 9,438 & 285 &, 000 & 69,9 \\
\hline & $q 18$ & $\begin{array}{l}\text { J'ai I'ambition d'exercer des fonctions importantes } \\
\text { grâce à mon engagement en politique. }\end{array}$ & 5,12 & 2,242 & 8,466 & 285 &, 000 & 66,4 \\
\hline & $q 19$ & $\begin{array}{l}\text { Je me sentirais encore plus utile en tant qu'acteur } \\
\text { politique. }\end{array}$ & 5,06 & 2,194 & 8,166 & 285 & ,000 & 65,7 \\
\hline & $q 20$ & $\begin{array}{l}\text { À travers le militantisme politique, j'ai de bonnes } \\
\text { chances de réaliser mes projets. }\end{array}$ & 4,98 & 2,094 & 7,906 & 285 & ,000 & 67,8 \\
\hline
\end{tabular}

Seuls 14,6 \% des enseignants ayant participé à l'enquête avouent se contenter d'enseigner, sans intérêt aucun pour les postes de nomination. Autrement, pour plus de $80 \%$ de l'échantillon, cet intérêt est avéré. De ce fait, la réussite d'une carrière tient également de l'occupation de postes de responsabilités dans la hiérarchie administrative. En réalité, ils confèrent honneurs, ressources financières et de multiples avantages sociaux que convoitent ceux qui n'occupent aucun poste de responsabilité. Plus des trois quarts de l'échantillon adhérent en effet à la proposition qui présente les collègues nommés aux postes convoités comme les plus épanouis au Cameroun. L'intérêt pour les postes de nomination est mesuré par cinq items. Pour un répondant qui exprime en permanence son désaccord entier, le score marqué est 5 alors qu'un pair qui est entièrement d'accord avec toutes les propositions fait un score de 35 . Pour un répondant neutre qui se situe à 4 pour chaque item, son score de neutralité totale est de 20 . Il s'agit de la moyenne hypothétique à partir de laquelle le test d'hypothèse portant sur une moyenne est fait. Sur cette considération, il est à relever que les scores échantillonnaux d'intérêt pour les postes de nomination sont significatifs chez les enseignants du secondaire étant donné que leur moyenne est remarquablement supérieure à $20 \quad\left[\bar{X}=24,94 ; s=5,433 ; \mu_{0}=20 ; t=15,085 ; d d l=274 ; p=, 000\right.$ $\left.\bar{X}=24,94 ; s=5,433 ; \mu_{0}=20 ; t=15,085 ; d d l=274 ; p=, 000\right]$. 
À l'item 6, une liste de postes de responsabilité dans l'enseignement secondaire a été proposée aux enseignants afin qu'ils indiquent s'ils y aspirent dans le cadre de leur carrière. Les pourcentages obtenus sont les suivants : 1) Animateur pédagogique (3,1\%); 2) Surveillant (2,1\%); 3) Surveillant général (6,3 \%); 4) Censeur (16,7\%); 5) Intendant (11,1\%); 6) Proviseur (41,1 \%); 7) Délégué départemental $(11,1 \%) ; 8)$ Délégué régional $(10,8 \%) ; 9)$ Inspecteur départemental de pédagogie (4,9\%); 10) Inspecteur national de pédagogie $(11,1 \%) ; 11)$ Chef de service au ministère $(6,6 \%)$; 12) Chef de bureau au ministère $(3,5 \%)$; 13) Cadre à l'Office du baccalauréat (7,7 \%); 14) Directeur à l'Office du baccalauréat $(8,7 \%)$; 15) Sous-directeur au ministère $(5,6 \%) ; 16)$ Directeur au ministère $(1,1 \%)$; 17) Secrétaire d'État à l'éducation (9,1\%); et enfin 18) Ministre des Enseignements secondaires $(11,1 \%)$. Le poste de proviseur semble celui qui suscite le plus d'aspirations. Il correspond à celui de chef d'établissement au sujet duquel des enquêtes ont déjà suggéré qu'il était le plus convoité.

Concernant l'intérêt pour les détachements dans les administrations autres que le ministère des Enseignements secondaires, plus de $78 \%$ des répondants avouent qu'ils se réjouiraient si une telle opportunité leur échouait en partage. Davantage, plus de $88 \%$, se disent prêts à accepter une telle offre, et ce, d'autant plus qu'ils estiment que les enseignants détachés dans d'autres administrations sont plus épanouis que leurs collègues qui « tiennent la craie » : il s'agit d'une image pour parler de ceux qui, en dehors de dispenser des cours, n'occupent aucun poste de responsabilité officiel dans l'administration. La justification avancée par plus de $90 \%$ tient de ce que les détachements dans d'autres administrations ont tout l'air de véritables promotions. Les scores moyens en matière d'intérêt pour le détachement sont tout aussi significatifs $\left[\bar{X}=21,67 ; s=4,146 ; \mu_{0}=16 ; t=15,085 ; d d l=271 ; p=, 000\right.$ $\left.\bar{X}=21,67 ; s=4,146 ; \mu_{0}=16 ; t=15,085 ; d d l=271 ; p=, 000\right]$.

Une question ouverte (item 11) invite les répondants à faire, tout en les justifiant, des propositions d'administrations dans lesquelles ils souhaiteraient être détachés. Parmi les départements ministériels les plus sollicités figurent : les Finances, l'Agriculture et le Développement rural, la Communication, le Plan et Aménagement du territoire, les Marchés publics, la Santé, les Postes et Télécommunications, les Travaux publics, la Jeunesse et Éducation civique, l'Enseignement supérieur, le Tourisme, la Recherche scientifique, les Eaux et Forêts, les Transports. Des administrations telles que la Présidence de la République, la Primature et le Sénat sont sollicitées. La Société nationale de raffinage (SONARA) est indiquée par un répondant.

Quant aux raisons les plus évoquées, en guise de justification, les enquêtés parlent surtout d'argent, d'honneur, d'avantages, de service divers et, dans quelques cas, de vocation. Quelques arguments choisis pour les illustrer sont : "la craie n'a aucun avantage, pas de mission, pas de primes", "les cadres de ces ministères sont respectés", "les enseignants détachés sont au moins des chefs de services dans les autres ministères; donc, ils ont plus d'avantages ", "Les finances parce que l'argent est à tout moment présent dans ce ministère et le pourboire est nombreux", "les missions existent, les cadres sont mieux traités ", "les primes trimestrielles sont élevées ailleurs », "tout travailleur a besoin d'être bien rémunéré». Il est opportun de relever que certains enseignants avancent d'autres raisons n'ayant rien à voir, en apparence, avec les motivations ci-devant citées. Ils parlent par exemple de vivre d'autres expériences professionnelles que celles de l'enseignant, ou alors de la valorisation de compétences et de formations acquises antérieurement. 
La détermination à créer des sources de revenus autres que le salaire est l'apanage de près de $94 \%$ des enseignants enquêtés. Plus de $90 \%$ envisagent d'ailleurs de monter des projets qui emploient plusieurs personnes. Àcet effet, une réussite en affaires pourrait amener près de $72 \%$ des répondantsà renoncer àleur carrière d'enseignant.En définitive, l'aspiration à gagner del'argent et à mener un train devie décent semble plus valorisée quel'exercice de la profession d'enseignant. Les scores d'ambitions d'hommes d'affaires sont tout aussi significatifs $\left[\bar{X}=22,15 ; s=3,981 ; \mu_{0}=16 ; t=25,272 ; d d l=267 ; p=, 000\right.$ $\left.\bar{X}=22,15 ; s=3,981 ; \mu_{0}=16 ; t=25,272 ; d d l=267 ; p=, 000\right]$. Une étude récente a d'ailleurs montré que l'un des éléments saillants de la représentation de l'emploi salarié publique est son offre d'opportunités de s'enrichir, en se servant dans les lignes de crédit, lorsqu'on est nommé à des postes de responsabilités importants (Njengoué Ngamaleu et Nkelzok Komtsindi, 2017).

L'intérêt pour la politique est une réalité avouée par plus de $71 \%$ des enseignants. Ils aspirent à faire partie des élites de leur localité par leur militantisme politique. C'est un moyen d'exercer des fonctions importantes en guise de compensation de leur engagement politique. Pour près de $66 \%$ des répondants, le sentiment d'être plus utile en tant qu'acteur politique plutôt qu'enseignant du secondaire ne fait pas l'ombre d'un doute. La perception de l'engagement en politique est de nature à favoriser des attentes de résultats plus valorisant que ce à quoi leur donne droit une simple carrière d'enseignant. Pour près de $68 \%$, les chances de réaliser leurs projets sont plus grandes à travers une implication en politique. Les scores relatifs à l'intérêt pour la politique comme moyen d'ascension socioprofessionnelle sont aussi significatifs que les précédents $\left[\bar{X}=25,88 ; s=10,147 ; \mu_{0}=20 ; t=9,627 ; d d l=275 ; p=, 000\right.$ $\left.\bar{X}=25,88 ; s=10,147 ; \mu_{0}=20 ; t=9,627 ; d d l=275 ; p=, 000\right]$.

\section{Stratégies de développement de carrière}

\section{Tableau 3}

Dimensions des stratégies de développement de carrière.

\begin{tabular}{|c|c|c|c|c|c|c|c|c|}
\hline & Item & & Moy & ET & $\mathrm{t}$ & ddl & $\mathrm{p}$ & Adhésion (\%) \\
\hline \multirow{4}{*}{ 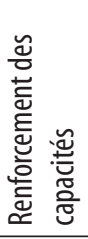 } & $\mathrm{q} 21$ & Je mets en permanence à jour mes connaissances. & 5,88 & ,914 & 34,815 & 285 &, 000 & 96,9 \\
\hline & $\mathrm{q} 22$ & Je poursuis mes études. & 5,40 & 1,415 & 16,717 & 285 &, 000 & 86,7 \\
\hline & q23 & Je fais des formations parallèles. & 5,31 & 1,435 & 15,411 & 285 &, 000 & 86,4 \\
\hline & q24 & $\begin{array}{l}\text { Je ne manque pas les occasions de recyclage (séminaires, } \\
\text { ateliers, stage, etc.) }\end{array}$ & 5,65 & 1,055 & 26,394 & 285 &, 000 & 94,1 \\
\hline \multirow{10}{*}{ 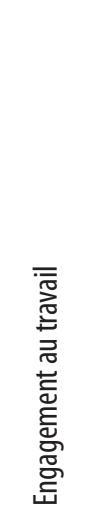 } & $\mathrm{q} 25$ & J'enseigne pour être le meilleur parmi ceux de ma discipline. & 5,54 & 1,127 & 23,049 & 282 &, 000 & 92,6 \\
\hline & $\mathrm{q} 26$ & Je me fais distinguer par mon travail. & 5,40 & 1,073 & 21,981 & 285 &, 000 & 82,5 \\
\hline & $\mathrm{q} 27$ & Je me fais remarquer par mon intégrité. & 5,45 & 1,044 & 23,446 & 285 &, 000 & 85,0 \\
\hline & $\mathrm{q} 28$ & Je respecte scrupuleusement ma hiérarchie. & 5,58 & 1,011 & 26,487 & 285 &, 000 & 85,3 \\
\hline & $\mathrm{q} 29$ & Je donne toujours au travail plus que ce qu'on attend de moi. & 5,39 & 1,066 & 21,954 & 281 &, 000 & 87,2 \\
\hline & $\mathrm{q} 30$ & Je suis rarement absent. & 5,51 & 1,018 & 25,147 & 285 &, 000 & 88,1 \\
\hline & q31 & Je suis ponctuel. & 5,53 & 968 & 26,812 & 285 &, 000 & 89,5 \\
\hline & $\mathrm{q} 32$ & J'assure toujours une excellente couverture des programmes. & 5,70 & ,901 & 31,729 & 283 &, 000 & 90,1 \\
\hline & q33 & J'évalue et corrige de manière irréprochable. & 5,74 & 913 & 32,205 & 285 &, 000 & 89,9 \\
\hline & $\mathrm{q} 34$ & Je recherche de bons bulletins de notes. & 5,38 & 1,277 & 18,272 & 284 &, 000 & 81,8 \\
\hline
\end{tabular}




\begin{tabular}{|c|c|c|c|c|c|c|c|c|}
\hline \multirow{4}{*}{ 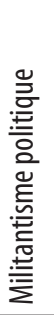 } & $\mathrm{q} 35$ & Je m’implique dans les activités politiques de ma localité. & 5,00 & 2,243 & 7,500 & 284 & 000 & 65,6 \\
\hline & q36 & $\begin{array}{l}\text { Je côtoie les hommes politiques capables de se souvenir de } \\
\text { moi. }\end{array}$ & 5,08 & 2,309 & 7,927 & 284 &, 000 & 66,7 \\
\hline & q37 & $\begin{array}{l}\text { Je pose des actes dans les milieux de la politique qui puissent } \\
\text { me faire remarquer. }\end{array}$ & 5,04 & 2,327 & 7,561 & 284 &, 000 & 65,3 \\
\hline & q38 & $\begin{array}{l}\text { Je me lance en politique avec l'intention de briguer des } \\
\text { fonctions importantes. }\end{array}$ & 4,93 & 2,300 & 6,771 & 280 &, 000 & 64,8 \\
\hline \multirow{5}{*}{ 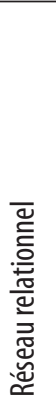 } & q39 & $\begin{array}{l}\text { Je m'arrange à avoir des relations sur qui je peux compter à } \\
\text { tout moment. }\end{array}$ & 5,55 & 1,532 & 17,060 & 285 & ,000 & 85,0 \\
\hline & $q 40$ & Je mets l'argent en jeu pour être affecté où je veux. & 4,75 & 2,078 & 6,073 & 284 &, 000 & 64,9 \\
\hline & $q 41$ & $\begin{array}{l}\text { Je mets l'argent en jeu pour être nommé aux postes que je } \\
\text { convoite. }\end{array}$ & 4,51 & 2,143 & 4,055 & 285 &, 000 & 62,6 \\
\hline & $q 42$ & $\begin{array}{l}\text { Je mets mon intimité en jeu si c'est nécessaire pour faire } \\
\text { avancer ma carrière. }\end{array}$ & 4,28 & 2,212 & 2,119 & 283 & ,035 & 59,2 \\
\hline & q43 & $\begin{array}{l}\text { J'adhère aux associations où j'ai l'occasion de rencontrer des } \\
\text { personnes qui peuvent donner un coup de pouce à ma carrière. }\end{array}$ & 4,77 & 1,832 & 6,777 & 256 & ,000 & 73,9 \\
\hline
\end{tabular}

En dépit des aspirations socioprofessionnelles qui tendent à indiquer que les motivations des enseignants sont plutôt extrinsèques, il se trouve qu'ils actualisent leurs connaissances (près de $97 \%$ ), sachant que cette démarche est indispensable pour tout formateur. Ils sont près de $87 \%$ qui disent poursuivre leurs études, ce qui est raisonnable étant donné que la plupart des répondants sont en service dans la ville de Yaoundé ou dans ses environs. Cela leur permet de fréquenter l'université avec plus d'aisance que ne le ferait un collègue qui vit et travaille dans une zone désenclavée, à des centaines de kilomètres des villes universitaires. C'est d'ailleurs la raison pour laquelle un peu plus de 86,4 \% avouent suivre des formations parallèles à l'exercice de leur profession d'enseignant du secondaire. Dans la même logique, ils saisissent toutes les occasions qui se présentent à eux pour bénéficier de l'organisation des séminaires, des ateliers, des stages, etc. Ce constat fait, les scores de renforcement de capacité sont significativement supérieurs à la moyenne hypothétique $\left[\bar{X}=22,24 ; s=3,462 ; \mu_{0}=16 ; t=29,954 ; d d l=275 ; p=, 000\right.$ $\left.\bar{X}=22,24 ; s=3,462 ; \mu_{0}=16 ; t=29,954 ; d d l=275 ; p=, 000\right]$.

Les différents items qui mesurent l'engagement au travail permettent d'enregistrer des scores très élevés, lesquels suscitent moult questionnements tant ils semblent contredire ceux des aspirations socioprofessionnelles. En effet, près de $93 \%$ des répondants prétendent qu'ils enseignent malgré tout pour être parmi les meilleurs de la discipline concernée. Ils disent d'ailleurs se distinguer par leur travail et se font remarquer également par leur intégrité. Ils donneraient au travail plus que ce qu'on attend effectivement d'eux, assurent une couverture des programmes convenable, évaluent les apprenants et corrigent dûment les copies, sont rarement absents, s'avèrent ponctuels, respectent la hiérarchie de la part de laquelle ils quêtent de bonnes notes, en termes d'évaluation et d'appréciation administratives. La question est de savoir pourquoi ils sont fascinés par les détachements dans d'autres administrations malgré cette dévotion béate au travail. Quoi qu'il en soit, force est de constater que les scores en matière d'engagement sont statistiquement significatifs $\quad\left[\bar{X}=55,22 ; s=6,049 ; \mu_{0}=40 ; t=41,043 ; d d l=265 ; p=, 000\right.$ $\left.\bar{X}=55,22 ; s=6,049 ; \mu_{0}=40 ; t=41,043 ; d d l=265 ; p=, 000\right]$. 
Les proportions d'adhésion aux propositions concernant le militantisme politique sont moins grandes que celles qui portent sur l'implication au travail certes, mais elles demeurent somme toute importantes $\mathrm{du}$ fait qu'elles concernent pour chacune d'elles plus de $64 \%$ de l'échantillon. Le militantisme politique renvoie pour les enseignants à l'implication dans les activités politiques de leur localité, les accointances avec les hommes politiques exerçant un certain pouvoir dont ils peuvent tirer profit en faveur de leur carrière. C'est sans doute la raison pour laquelle plus de $65 \%$ des répondants avouent poser des actes qui les fassent remarquer dans les milieux politiques, et ce, d'autant plus qu'ils s'engagent en politique avec l'intention de briguer des fonctions importantes. Les scores en matière de militantisme politique sont à toutlemoinssignificatifs $\left[\bar{X}=20,47 ; s=8,842 ; \mu_{0}=16 ; t=22,567 ; d d l=271 ; p=, 000\right.$ $\left.\bar{X}=20,47 ; s=8,842 ; \mu_{0}=16 ; t=22,567 ; d d l=271 ; p=, 000\right]$.

La psychologie des relations sociales étant de nature à favoriser l'ascension socioprofessionnelle (Njengoué Ngamaleu, 2015), les répondants avouent, pour $85 \%$, rechercher des relations sur lesquelles compter à tout moment. Près de $65 \%$ affirment mettre de l'argent en jeu pour être affectés où ils souhaitent travailler. Il a été démontré dans l'étude précédemment citée qu'il ne suffit pas de disposer de l'argent à cet effet, encore faut-il savoir à qui le remettre pour ne pas le donner en vain. En cela s'avère importante l'habileté à dénicher les personnes effectivement capables de mener à l'aboutissement de telles initiatives. C'est la réalité socioculturelle $\mathrm{du}$ «bon réseau ». L'enjeu de l'argent misé sert dans la même logique à l'accès aux postes de responsabilité par voie de nomination (62,6 \%). Près de $60 \%$ avouent «mettre leur intimité en jeu si c'est nécessaire pour faire avancer leur carrière ». Les implications de cet aveu sont multiples et remettent en question dans de tout autres perspectives la morale individuelle et le prix à payer, en termes de dignité, pour arriver à ses fins. Que signifie pour une femme mettre en jeu son intimité pour favoriser sa carrière? Que signifie la même chose pour un homme? Il peut être épilogué sur cette préoccupation sans en finir, tellement elle révèlerait des anecdotes des plus ondoyantes et diverses. Toutefois, pour près de $74 \%$ des répondants, l'adhésion aux associations est aussi motivée par la possibilité qu'elles offrent de rencontrer des personnes qui peuvent contribuer à l'embellissement de leur carrière. Les scores en termes de réseau relationnel sont statistiquement significatifs

$$
\left[\overline{\mathrm{X}}=23,73 ; \mathrm{s}=8,674 ; \mu_{0}=20 ; \mathrm{t}=6,732 ; \mathrm{ddl}=244 ; \mathrm{p}=, 000\right.
$$
$\left.\bar{X}=23,73 ; s=8,674 ; \mu_{0}=20 ; t=6,732 ; \mathrm{ddl}=244 ; \mathrm{p}=, 000\right]$.

Les stratégies de développement de carrière sont diversifiées, et les unes aussi importantes que les autres. Essayer de renforcer en permanence ses capacités et bien faire son travail semblent compter au même titre que militer en politique ou recourir aux relations sociales influentes. Il devient intéressant d'examiner les corrélations entre les différentes dimensions mesurées. Le tableau 4 y renseigne en tant que matrice de corrélations.

Toutes les dimensions des aspirations socioprofessionnelles sont significativement corrélées entre elles. La matrice de corrélations indique quelles le sont à un seuil critique de 0,01. Lintérêt pour les nominations, l'intérêt pour le détachement, les ambitions relatives aux affaires et l'intérêt pour la politique ont les unes avec les autres une relation positive. La corrélation la plus forte est celle qui décrit la relation entre l'intérêt pour la politique et les ambitions d'affaires $(r=0,506 ; p<, 01)$.

Pour ce qui concerne les modalités des stratégies de développement de carrière, elles sont certes significativement corrélées entre elles, mais dans une tout autre configuration que celle qui concerne les modalités des aspirations socioprofessionnelles. En effet, certaines sont négativement corrélées entre elles tandis que d'autres le sont positivement. Le renforcement des capacités est positivement corrélé à l'implication au travail $(\mathrm{r}=0,228 ; \mathrm{p}<, 01)$, mais bien moins qu'au militantisme politique $(\mathrm{r}=0,436$; 
$\mathrm{p}<, 01)$ et au réseau relationnel $(\mathrm{r}=0,438 ; \mathrm{p}<, 01)$. Justement, l'implication au travail attire l'attention parce qu'elle est négativement corrélée au réseau relationnel $(\mathrm{r}=-0,217 ; \mathrm{p}<, 01)$ et au militantisme politique $(\mathrm{r}=-0,184 ; \mathrm{p}<, 01)$. La relation est positive et forte entre la stratégie consistant à militer en politique et celle qui consiste à recourir au réseau relationnel $(\mathrm{r}=0,876 ; \mathrm{p}<, 01)$.

S'agissant des relations entre les aspirations socioprofessionnelles et les stratégies de développement de carrière, l'intérêt pour les nominations est positivement corrélé aux quatre stratégies envisagées : toutefois, le lien est plus fort avec le militantisme politique $(\mathrm{r}=0,350 ; \mathrm{p}<, 01)$ et le réseau relationnel $(\mathrm{r}=0,332 ; \mathrm{p}<, 01)$ qu'avec les deux autres que sont le renforcement de capacités $(\mathrm{r}=0,209 ; \mathrm{p}<, 01)$ et l'implication au travail $(\mathrm{r}=0,217 ; \mathrm{p}<, 01)$.

Concernant l'intérêt pour le détachement, cette aspiration socioprofessionnelle n'est pas du tout corrélée à l'implication au travail. Elle est par contre mieux corrélée au militantisme politique $(r=0,384$; $\mathrm{p}<, 01)$ et au réseau relationnel $(\mathrm{r}=0,466 ; \mathrm{p}<, 01)$ qu'au renforcement de capacités. Cette tendance est confirmée par la troisième forme d'aspirations socioprofessionnelles, à savoir les ambitions d'affaires. Elles n'ont aucun lien avec l'implication au travail et s'avèrent plus liées au réseau relationnel $(\mathrm{r}=0,529 ; \mathrm{p}<, 01)$ et au militantisme politique $(\mathrm{r}=0,513 ; \mathrm{p}<, 01)$ qu'au renforcement de capacités $(\mathrm{r}=0,271 ; \mathrm{p}<, 01)$.

Quant aux ambitions politiques, elles sont négativement corrélées à l'implication au travail $(\mathrm{r}=$ $0,167 ; \mathrm{p}<, 01)$. Autrement dit, plus les ambitions politiques sont élevées, moins l'implication au travail semble pertinente comme stratégie de développement de carrière. Par contre, le lien avec le renforcement des capacités est non seulement positif, mais plus fort $(r=0,442 ; p<, 01)$. Ce lien est davantage considérable avec les stratégies que sont le réseau relationnel $(\mathrm{r}=0,840 ; \mathrm{p}<, 01)$ et surtout le militantisme politique $(\mathrm{r}=0,928 ; \mathrm{p}<, 01)$. 
Tableau 4

Matrice de corrélations.

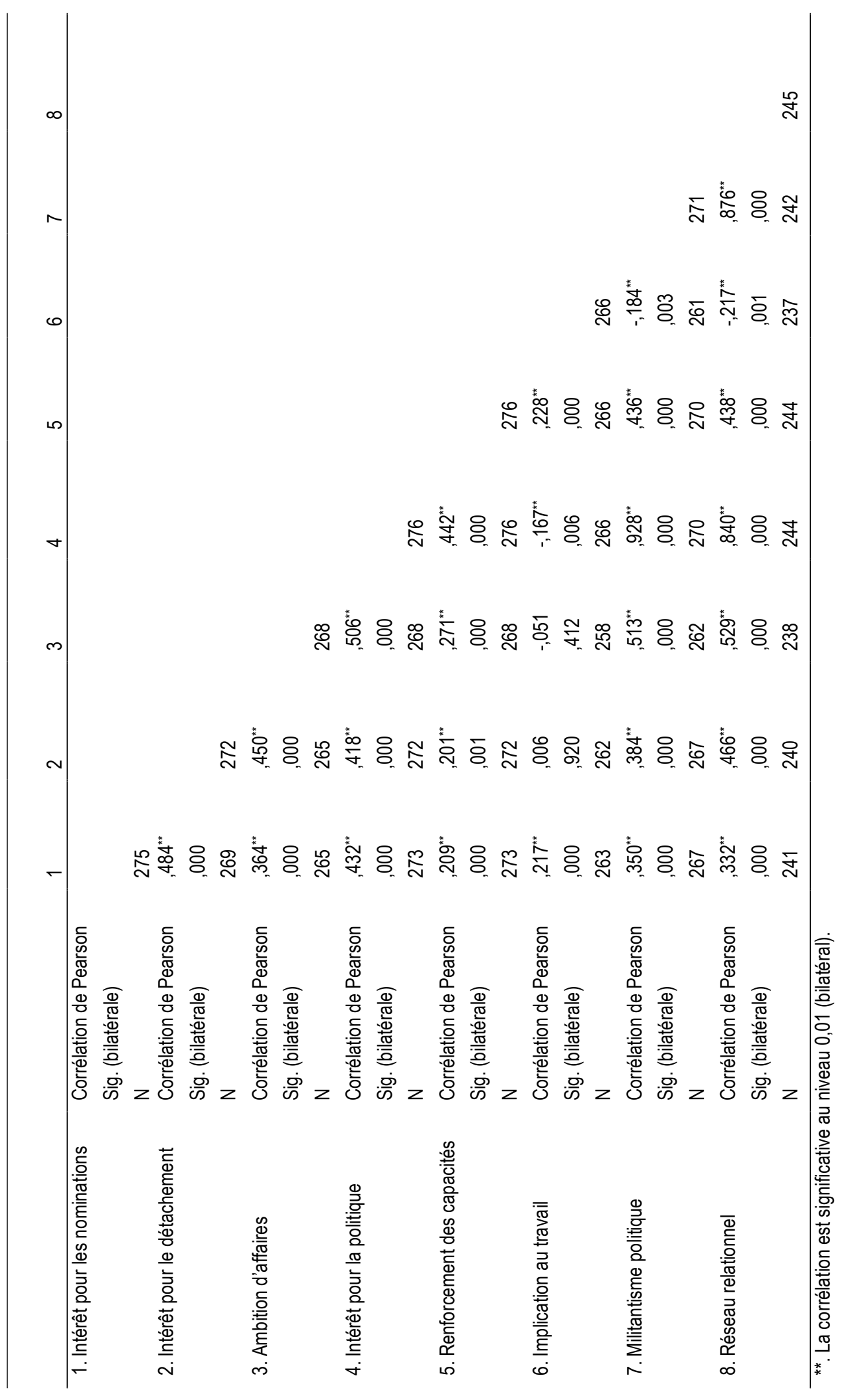




\section{Discussion et conclusion}

Nous avons mesuré les aspirations socioprofessionnelles d'enseignants du secondaire au Cameroun et les stratégies de développement de carrière qui s'y rapportent. Il s'agit, pour les premières, de l'intérêt pour les postes de nomination, l'intérêt pour le détachement dans d'autres administrations, les ambitions d'affaires et l'intérêt pour une carrière en politique; et, pour les secondes, du renforcement des capacités, de l'implication au travail, du militantisme politique et du réseau relationnel. Ces modalités sont choisies en fonction du contexte managérial des ressources humaines de l'enseignement secondaire, caractérisé par des dysfonctionnements qui laissent libre cours aux pratiques non conformes aux textes réglementaires.

Le grand paradoxe des résultats de cette étude réside dans le fait que les professionnels, formés à un métier et destinés à l'exercer, considèrent qu'ils sont mieux gratifiés et privilégiés une fois insérés dans d'autres administrations, pour s'occuper au quotidien de tâches en rupture avec la pratique de l'enseignement ou des activités et fonctions s'y rapportant. Le choix initial de la profession d'enseignant semble celui dont on se contente en attendant de trouver de meilleures occupations professionnelles, ou alors mieux qui puisse procurer plus d'honneurs, de prestige, de privilèges sociaux et un pouvoir d'achat satisfaisant.

Plus l'implication au travail est valorisée comme stratégie de développement de carrière, moins les enseignants ont recours au militantisme politique et au réseau relationnel. Le renforcement des capacités n'exclut ni le militantisme politique ni le recours au réseau relationnel, alors que ce n'est pas le cas pour l'implication au travail. Le renforcement des capacités et l'implication au travail sont des stratégies qui semblent moins garantes de promotions, à travers les nominations, que le militantisme politique et le réseau relationnel. Autrement dit, pour espérer bénéficier d'un détachement dans une autre administration, l'enseignant du secondaire s'appuie plus sur son entregent et son activisme en politique. Le militantisme politique et le réseau relationnel sont également favorables à la réalisation des ambitions d'affaires. Par ailleurs, lorsque les ambitions politiques sont affirmées, la stratégie idéale pour les réaliser n'est ni plus ni moins que le militantisme politique. Toutefois, le renforcement des capacités sert aussi l'ambition politique. Faire preuve de compétences pourrait être un atout en politique.

Les aspirations socioprofessionnelles des enseignants du secondaire au Cameroun sous-tendent des buts et des attentes de résultats qui ont trait aux croyances personnelles relatives aux conséquences et aux résultats de la réalisation de comportements particuliers. D'après Lent (2008), les valeurs relatives à la vie professionnelle sont construites dans le cadre du concept des attentes de résultats. Il précise que ces attentes de résultats sont une combinaison de préférences des personnes pour des caractéristiques professionnelles particulières ou pour des renforçateurs (le statut, l'argent, l'autonomie) et de croyances relatives au fait de savoir jusqu'à quel point ces professions particulières offrent bien ces avantages.

Les enseignants recherchent ces renforçateurs dans l'occupation des postes de nomination, à travers le détachement dans d'autres administrations (les ministères des Finances ou des Marchés publics, par exemple), dans la possibilité de faire des affaires, à travers une implication en politique ou la recherche d'un réseau relationnel influent. Le contexte camerounais leur permet aisément d'orienter leur devenir professionnel hors du cadre de l'enseignement secondaire. Ces aspirations se muent en véritables buts personnels, à savoir des intentions de s'engager dans une activité précise pour atteindre un objectif 
particulier (Bandura, 1986). En cela interviennent les stratégies de développement de carrière. Le contexte managérial et administratif pèse de son poids en influant sur les capacités des enseignants ou sur leur volonté de traduire leurs intérêts en buts et leurs buts en actions. Ceci est d'ailleurs fidèle à la pensée de Lent (2008) qui estime que les intérêts professionnels ont une probabilité plus grande de devenir des buts, lesquels à leur tour ont une probabilité plus grande d'être atteints, quand les personnes rencontrent, à l'égard de leurs projets professionnels, de forts soutiens environnementaux et des obstacles surmontables.

En ce qui concerne le développement de carrière des enseignements du secondaire au Cameroun, les soutiens environnementaux en question relèvent en grande partie de la malgouvernance des ressources humaines. Le constat selon lequel l'autodétermination des professionnels d'un corps de métier doive transiger à la fois avec les règles formelles, au pouvoir régulateur affaibli, et des pratiques informelles, prévalant de manière importante, amène à poser le problème de l'agentivité humaine dans des contextes d'incertitude. L'agentivité relevant d'une psychologie positive, il est tout indiqué de poser le problème scientifique de l'autodétermination en Afrique noire en général et au Cameroun, en particulier, où le pouvoir régulateur des normes informelles fait une concurrence, parfois victorieuse, aux normes formelles au pouvoir régulateur fragilisé. Comme c'est le cas avec les enseignants du secondaire, les renforcements sont alors délivrés par des opportunités et des buts, qui profitent certes à quelques individus, mais compromettent les performances du système dans lequel ils évoluent.

\section{Références}

Bandura, A. (1986). Social foundations of thought and action. Englewood Cliffs, NJ: Prentice- Hall.

Bandura, A. (1977). Social learning theory. Englewood Cliffs, NJ : Prentice-Hall.

Bandura, A. (1989). Human agency in social cognitive theory. American Psychologist, 44(9), 1175-1184. http://dx.doi. org/10.1037/0003-066X.44.9.1175

Bandura, A. (1994). Self-efficacy. Dans V. S. Ramachaudran (dir.), Encyclopedia of human behavior (vol. 4, p. 71-81). Repéré à http://www.uky.edu/ eushe2/Bandura/BanEncy.html

Bandura, A. (1997). Self-efficacy: The exercise of control. New York, NY : W. H. Freeman.

Bandura, A. (2003). Auto-efficacité: le sentiment d'efficacité personnelle. Paris : De Boeck Université.

Blundo, G., van Beek, W. E. A. (2002). Synthèse de l'atelier « Corruption et bureaucratie ». Bulletin de l'APAD, (23-24).

Commission nationale anti-corruption (CONAC). (2010). Stratégie nationale de lutte contre la corruption 2010-2015.

Commission nationale anti-corruption (CONAC). (2011). Rapport sur l'état de la lutte contre la corruption au Cameroun. Repéré à https://www.acauthorities.org/sites/aca/files/countrydoc/Republique\%20Du\%20Cameroun\%20Paix\%20\% E2\%80\%93\%20Travai1\%20\%E2\%80\%93\%20Patrie\%20.pdf

Djateng, F. (dir.) (2012). La corruption dans le secteur éducatif. Pratiques et faits, mécanisme de lutte dans le secteur de l'éducation secondaire: le cas de la région de l'Ouest Cameroun (Document de travail). Bafoussam, Cameroun.

Friedrich-Ebert-Stiftung, (2015). La corruption pervertit-elle le système éducatif au Cameroun ? Repéré à http://www.feskamerun.org/cameroon/index.php/activities/113-la-corruption-pervertit-elle-le-systeme-educatif-au-cameroun

Guichard, J. et Huteau, M. (2007). Orientation et insertion professionnelle : 75 concepts clés. Paris : Dunod.

Lent, R.W. (2005). A social cognitive view of career development and counseling. Dans S. D. Brown et R. W. Lent (dir.), Career development and counselling: putting theory and research to work (p. 101-127). Hoboken, NJ : Wiley. 
Lent, R. W. (2008). Une conception sociale cognitive de l'orientation scolaire et professionnelle : considérations théoriques et pratiques. L'orientation scolaire et professionnelle, 37(1), 57-90. http://dx.doi.org/10.4000/osp.1597

Lent, R. W., Brown, S. D. et Hackett, G. (1994). Toward a unifying social cognitive theory of career and academic interest, choice, and performance. Journal of Vocational Behavior, 45(1), 79-122. http://dx.doi.org/10.1006/jvbe.1994.1027

Lent, R. W., Brown, S. D. et Hackett, G. (2000). Contextual supports and barriers to career choice: a social cognitive analysis. Journal of Counseling Psychology, 47(1), 36-49. http://dx.doi.org/10.1037//0022-0167.47.1.36

Litalien, D. et Guay, F. (2010). Validation d'un modèle motivationnel des aspirations professionnelles. Revue canadienne de l'éducation, 33(4), 732-760. Repéré à http://journals.sfu.ca/cje/index.php/cje-rce/article/view/2192/1818

Ndongmo, M. et Bayang, D. (2015). Comment pouvons-nous envisager une lutte préventive pour une société camerounaise plus juste? Débarrassons nos écoles de la corruption. Repéré à http://gredevel.fr/phocadownload/publications/Luttons\%20co ntre\%201a\%20corruption.pdf

Ngon, O. (2015). Cameroun - Éducation : Un réseau de vente des postes de « Proviseurs de Lycées » démantelé. Repéré à http://www.cameroon-info.net/article/cameroun-education-un-reseau-de-vente-des-postes-de-proviseur-de-lyceesdemantele-246006.html

Njengoué Ngamaleu, H. R. (2015). Concours administratifs et psychologie des relations sociales au Cameroun. Revue Perspectives et société, 6(1), 30-47.

Njengoué Ngamaleu, H. R. et Nkelzok Komtsindi, V. (2017). Prestige de la fonction publique camerounaise et attitude des étudiants vis-à-vis de l'entrepreneuriat. Revue Sociétés et Économies, (11), 164-184.

O’Brien, K. M. (1996). The influence of psychological separation and parental attachment on the career development of adolescent women. Journal of Vocational Behavior, 48(3), 257-274. http://dx.doi.org/10.1006/jvbe.1996.0024

Omgba, J.-F. (2015). Pouvoir discrétionnaire des acteurs de première ligne en contexte de développement: analyse des stratégies d'enseignants dans l'accessibilisation de l'éducation secondaire au Cameroun (Thèse de doctorat, Université du Québec). Repéré à http://archives.enap.ca/bibliotheques/2015/08/030889225.pdf

Rasheed Ali, S. et Saunders, J. L. (2008). The career aspirations of rural Appalachian high school students. Journal of Career Assessment, 17(2), 172-188. http://dx.doi.org/10.1177/1069072708328897

Titi Nwel, P. (1999). De la corruption au Cameroun. Repéré à https://library.fes.de/pdf-files/bueros/kamerun/07798.pdf

Titi Nwel, P. (2009). La lutte contre la corruption au Cameroun de 1999 à 2008. Yaoundé : Presses universitaires d'Afrique.

Transparency International. (2007). Système national d'intégrité. Yaoundé : Transparency International.

Young, R. A., Marshall, S. K. et Valach, L. (2007). Making career theories more culturally sensitive: Implications for counseling. The Career Development Quarterly, 56(1), 4-18. http://dx.doi.org/10.1002/j.2161-0045.2007. $\underline{\text { tb00016.x }}$

Young, R. A. et Valach, L. (2000). Reconceptualising career theory and research: An action-theoretical perspective. Dans A. Collin et R. A. Young (dir.), The future of career (p. 181-196). Cambridge : Cambridge University Press. http:// dx.doi.org/10.1017/cbo9780511520853.012

Young, R. A. et Valach, L. (2006). La notion de projet en psychologie de l'orientation. L'orientation scolaire et professionnelle, 35(4), 495-509. http://dx.doi.org/10.4000/osp.1168

\section{Pour citer cet article}

Njengoué Ngamaleu, H. R. et Dang Olinga, C. (2019). Aspirations socioprofessionnelles et stratégies de développement de carrière chez les enseignements du secondaire au Cameroun. Formation et profession. 27(2). 84-100.

http://dx.doi.org/10.18162/fp.2018.438 